\title{
SHIFTED CONVOLUTION SUMS INVOLVING THETA SERIES
}

\author{
QINGFENG SUN
}

\begin{abstract}
Let $f$ be a cuspidal newform (holomorphic or Maass) of arbitrary level and nebentypus and denote by $\lambda_{f}(n)$ its $n$-th Hecke eigenvalue. Let

$$
r(n)=\#\left\{\left(n_{1}, n_{2}\right) \in \mathbb{Z}^{2}: n_{1}^{2}+n_{2}^{2}=n\right\} .
$$
\end{abstract}

In this paper, we study the shifted convolution sum

$$
\mathcal{S}_{h}(X)=\sum_{n \leq X} \lambda_{f}(n+h) r(n), \quad 1 \leq h \leq X,
$$

and establish uniform bounds with respect to the shift $h$ for $\mathcal{S}_{h}(X)$.

\section{INTRODUCTION}

The shifted convolution sum

$$
\sum_{n \leq X} a_{1}(n+h) a_{2}(n)
$$

where $a_{1}(n)$ and $a_{2}(n)$ are two arithmetic functions and $h \geq 1$ an integer, is an interesting and important object in analytic number theory and has been studied intensively by many authors with various applications (see for example [2], [6], [11], [13], [15]). For the arithmetic function

$$
r_{\ell}(n)=\#\left\{\left(n_{1}, n_{2}, \ldots, n_{\ell}\right) \in \mathbb{Z}^{\ell}: n_{1}^{2}+\cdots+n_{\ell}^{2}=n\right\},
$$

which is the $n$-th Fourier coefficient of the modular form $\theta^{\ell}(z)$, where $\theta(z)$ is the classical Jacobi theta series

$$
\theta(z)=\sum_{n \in \mathbb{Z}} e\left(n^{2} z\right)
$$

the related shifted convolution problem was first studied by Luo [12]. Precisely, Luo first established a Voronoi formula for $r_{\ell}(n)$ and then applying Poincaré series reduction and his Voronoi formula proved the following: Let $\lambda_{g}(n)$ be the normalized $n$-th Fourier coefficient of a holomorphic cusp form $g$ of weight $\kappa$ for $\Gamma_{0}(N)$. For $\ell \geq 2$ and $\kappa \geq \frac{\ell}{2}+3$,

$$
\sum_{n \leq X} \lambda_{g}(n+h) r_{\ell}(n) \ll_{h, g, \ell, \varepsilon} X^{\frac{\ell}{2}-\vartheta_{\ell}+\varepsilon},
$$

where

$$
\vartheta_{\ell}=\frac{\ell-1}{4(\varrho+1)}, \quad \varrho= \begin{cases}\frac{1+\ell}{2}, & \text { if } \ell \text { odd }, \\ 1+\frac{\ell}{2}, & \text { if } \ell \text { even. }\end{cases}
$$

2010 Mathematics Subject Classification: 11F27, 11F30, 11F37.

Key words and phrases. Shifted convolution sum, cuspidal newform, theta series. 
In particular, $\vartheta_{2}=\frac{1}{12}, \vartheta_{3}=\frac{1}{6}$. Recently, Lü, Wu and Zhai [14] improved Luo's result by the circle method and showed that (1.1) holds uniformly for $0 \leq h \leq X$ and all $\kappa$, and $\vartheta_{\ell}$ can be taken as $\vartheta_{3}=\frac{1}{4}, \vartheta_{\ell}=\frac{1}{2}(\ell \geq 4)$. Moreover, for $N=1$, using some new ideas, they proved that one can take $\vartheta_{2}=\frac{1}{6}, \vartheta_{\ell}=\frac{2}{3}(\ell \geq 6)$. For other interesting results, see [16].

In this paper, we are concerned with the shifted convolution problem of theta series with a general $G L(2)$ cusp form, that is (as in [1]) a holomorphic form of integral weight $\kappa \geq 1$, level $D$ and nebentypus $\chi_{D}$, or a Maass form of weight 0 or 1 , level $D$, nebentypus $\chi_{D}$ and Laplace eigenvalue $1 / 4+\mu^{2}$. Suppose that $f$ is a newform and denote by $\lambda_{f}(n)$ its $n$-th Hecke eigenvalue. Then we have the following result.

Theorem 1. Let $f$ be a cuspidal newform (holomorphic or Maass) of arbitrary level and nebentypus and denote by $\lambda_{f}(n)$ its $n$-th Hecke eigenvalue. Assume that $\lambda_{f}(n) \ll n^{\theta+\varepsilon}$ for any $\varepsilon>0$. Then for $2 \leq \ell \leq 7$ and $X>1$, we have

$$
\sum_{n \leq X} \lambda_{f}(n+h) r_{\ell}(n) \ll_{f, \ell, \varepsilon} X^{\frac{\ell}{2}-\frac{\ell-1-2 \theta}{12}+\varepsilon}
$$

uniformly for $1 \leq h \leq X$.

The most interesting case of Theorem 1 is $\ell=2$. Note that the best $\theta$ we known is $\theta=7 / 64$ (see [10]). Denote $r_{2}(n):=r(n)$ as usual. By Theorem 1, we have

$$
\sum_{n \leq X} \lambda_{f}(n+h) r(n) \ll_{f, \varepsilon} X^{1-\frac{25}{384}+\varepsilon} .
$$

Remark 1. For $\ell \geq 3$, Theorem 1 is weaker than the results in [14]. In fact, the argument in Section 3 in [14] yields $\vartheta_{3}=\frac{1}{4}, \vartheta_{\ell}=\frac{1}{2}$ for $\ell \geq 4$.

To prove Theorem 1, we apply Jutila's variation of the circle method (see [9]) which gives an approximation for

$$
I_{[0,1]}(x)= \begin{cases}1, & x \in[0,1] \\ 0, & \text { otherwise. }\end{cases}
$$

Precisely, let $\mathcal{Q} \subset[1, Q], Q>0$ and $Q^{-2} \leq \delta \leq Q^{-1}$. Define

$$
\widetilde{I}_{\mathcal{Q}, \delta}(x)=\frac{1}{2 \delta L} \sum_{q \in \mathcal{Q}} \sum_{\substack{a=1 \\(a, q)=1}}^{q} I_{\left[\frac{a}{q}-\delta, \frac{a}{q}+\delta\right]}(x),
$$

where $L=\sum_{q \in \mathcal{Q}} \phi(q)$. Then $\widetilde{I}_{\mathcal{Q}, \delta}(x)$ is an approximation for $I_{[0,1]}(x)$ in the following sense (see Lemma 4 in Munshi [15]):

$$
\int_{0}^{1}\left|1-\widetilde{I}_{\mathcal{Q}, \delta}(\beta)\right|^{2} \mathrm{~d} \beta \ll \frac{Q^{2+\varepsilon}}{\delta L^{2}} .
$$

We shall prove Theorem 1 in detail in Section 3.

The advantage of Jutila's version of the circle method is the flexibility of choosing moduli and this is good for us to study a general $G L(2)$ cusp form. However, for our problem, there is some lost from (1.4). Thus, for $\ell=2$ and $f$ a holomorphic cusp form 
of weight $\kappa$ or a Maass cusp form of Laplace eigenvalue $\frac{1}{4}+\mu^{2}$ for $S L(2, \mathbb{Z})$, we further prove the following result.

Theorem 2. Let $f$ be a holomorphic cusp form of weight $\kappa$ or a Maass cusp form of Laplace eigenvalue $\frac{1}{4}+\mu^{2}$ for $S L(2, \mathbb{Z})$ and denote by $\lambda_{f}(n)$ its $n$-th Hecke eigenvalue. Let $\phi(x)$ be a smooth function which is supported on $[1 / 2,1]$ and identically equal to 1 on $\left[1 / 2+\Delta^{-1}, 1-\Delta^{-1}\right]$ with $\Delta>4$, satisfying $\phi^{(j)}(x) \ll_{j} \Delta^{j}$ for all integers $j \geq 0$. Then for $X>1$ and any $\varepsilon>0$, we have

$$
\sum_{n \geq 1} \lambda_{f}(n+h) r(n) \phi\left(\frac{n}{X}\right) \ll_{f, \varepsilon} X^{1-\frac{1}{4}+\varepsilon} \Delta
$$

uniformly for $1 \leq h \leq X$.

Remark 2. For holomorphic cusp forms, Theorem 2 improves the result in Luo [12], where it is proved that

$$
\sum_{n \geq 1} \lambda_{f}(n+h) r(n) \phi\left(\frac{n}{X}\right) \ll_{f, \varepsilon} X^{1-\frac{1}{4}+\varepsilon} \Delta^{2} .
$$

Assume that $\lambda_{f}(n) \ll n^{\theta+\varepsilon}$. By Theorem 2 we have

$\sum_{X / 2 \leq n \leq X} \lambda_{f}(n+h) r(n)=\sum_{n \geq 1} \lambda_{f}(n+h) r(n) \phi\left(\frac{n}{X}\right)+O_{\varepsilon}\left(X^{1+\theta+\varepsilon} \Delta^{-1}\right) \ll_{f, \varepsilon} X^{\frac{3}{4}+\varepsilon} \Delta+X^{1+\theta+\varepsilon} \Delta^{-1}$.

On taking $\Delta=X^{\frac{1}{8}+\frac{\theta}{2}}$, we obtain

$$
\sum_{X / 2 \leq n \leq X} \lambda_{f}(n+h) r(n) \ll_{f, \varepsilon} X^{1-\frac{1-4 \theta}{8}+\varepsilon},
$$

uniformly for $1 \leq h \leq X$. Thus we get the following result which improves (1.2).

Corollary 1. Let $f$ be a holomorphic cusp form of weight $\kappa$ or a Maass cusp form of Laplace eigenvalue $\frac{1}{4}+\mu^{2}$ for $S L(2, \mathbb{Z})$ and denote by $\lambda_{f}(n)$ its $n$-th Hecke eigenvalue. Assume that $\lambda_{f}(n) \ll n^{\theta+\varepsilon}$. Then for $X>1$ and any $\varepsilon>0$, we have

$$
\sum_{n \leq X} \lambda_{f}(n+h) r(n) \ll_{f, \varepsilon} X^{1-\frac{1-4 \theta}{8}+\varepsilon}
$$

uniformly for $1 \leq h \leq X$.

For the proof of Theorem 2, we use a different method from [12, [14] and Theorem 1. Precisely, as in [17], we apply the classical Hardy-Littlewood-Kloosterman circle method to transform the sum in Theorem 2. Then we can apply the Voronoi formula for the cusp form $f$ and an asymptotic formula for the sum $\sum_{|m| \leq \sqrt{X}} e\left(\alpha m^{2}\right), \alpha \in[0,1]$, to get essentially three sums involving transforms of Bessel functions and some exponential sums. Finally, Theorem 2 follows from nontrivial estimates of these transforms and exponential sums. 


\section{VORONOI FORMULAS}

We need Voronoi formulas for both $\lambda_{f}(n)$ and $r_{\ell}(n)$. Let $J_{s}, K_{s}$ and $Y_{s}$ denote the standard $J$-Bessel function, $K$-Bessel function and $Y$-Bessel function, respectively. Let $f$ be a general $G L(2)$ cusp form, that is (as in [1]) a holomorphic cusp form of integral weight $\kappa \geq 1$, level $D$ and nebentypus $\chi_{D}$, or a Maass cusp form of weight 0 or 1 , level $D$, nebentypus $\chi_{D}$ and Laplace eigenvalue $1 / 4+\mu^{2}$. Suppose that $f$ is a newform and denote by $\lambda_{f}(n)$ its $n$-th Hecke eigenvalue. Then we have the following Voronoi formula for $\lambda_{f}(n)$ (see [1] or [4, Proposition 2.1)

Lemma 1. Let $f$ be a cusp form (holomorphic or Maass) of weight $\kappa$, level $D$ and nebentypus $\chi_{D}$. Let $q \equiv 0 \bmod D$ and let a be an integer coprime to $q$ and $a d \equiv 1(\bmod q)$. If $v(x)$ is a compactly supported smooth function on $\mathbb{R}^{+}$, then

$$
\begin{aligned}
\sum_{n \geq 1} \lambda_{f}(n) e\left(\frac{a n}{q}\right) v(n)= & \frac{\chi_{D}(d)}{q} \sum_{n \geq 1} \lambda_{f}(n) e\left(-\frac{d n}{q}\right) \mathcal{V}^{+}\left(\frac{n}{q^{2}}\right) \\
& +\omega_{f} \frac{\Gamma\left(\frac{1}{2}+i \mu-\frac{\kappa}{2}\right)}{\Gamma\left(\frac{1}{2}+i \mu+\frac{\kappa}{2}\right)} \frac{\chi_{D}(d)}{q} \sum_{n \geq 1} \lambda_{f}(n) e\left(\frac{d n}{q}\right) \mathcal{V}^{-}\left(\frac{n}{q^{2}}\right),
\end{aligned}
$$

where $\omega_{f}$ is a constant depending only on $f, \mu$ is the spectral parameter of $f$ in the Maass case, and

$$
\mathcal{V}^{ \pm}(y)=\int_{0}^{\infty} v(x) \mathcal{H}_{f}^{ \pm}(4 \pi \sqrt{x y}) d x .
$$

In this formula, (i) if $f$ is induced from a holomorphic form of weight $k$,

$$
\mathcal{H}_{f}^{+}(x)=2 \pi i^{k} J_{k-1}(x), \quad \mathcal{H}_{f}^{-}(x)=0 ;
$$

(ii) if $\kappa$ is even, $f$ is not induced from a holomorphic form,

$$
\mathcal{H}_{f}^{+}(x)=\frac{-\pi}{\cosh (\pi \mu)}\left\{Y_{2 i \mu}(x)+Y_{-2 i \mu}(x)\right\}, \quad \mathcal{H}_{f}^{-}(x)=4 \cosh (\pi \mu) K_{2 i \mu}(x) ;
$$

(iii) if $\kappa$ is odd, $f$ is not induced from a holomorphic form,

$$
\mathcal{H}_{f}^{+}(x)=\frac{\pi}{\sinh (\pi \mu)}\left\{Y_{2 i \mu}(x)-Y_{-2 i \mu}(x)\right\}, \quad \mathcal{H}_{f}^{-}(x)=-4 i \sinh (\pi \mu) K_{2 i \mu}(x) .
$$

$\mathcal{V}^{ \pm}(y)$ has the following properties.

Lemma 2. If $v(x)$ is a smooth function supported on $[A Y, B Y](B>A>0)$ satisfying $x^{j} v^{(j)}(x) \ll_{A, B, j} 1$, then for any integer $j \geq 0$ and any $\varepsilon>0$,

$$
\mathcal{V}^{ \pm}(y) \ll_{f, A, B, j, \varepsilon} Y(1+\sqrt{y Y})^{-\frac{1}{2}}\left(1+\frac{1}{\sqrt{y Y}}\right)^{2|\operatorname{Im} \mu|+\varepsilon}\left(\frac{1}{\sqrt{y Y}}+\frac{1}{y Y}\right)^{j}
$$

where $\mu$ is the spectral parameter of $f$ in the Maass form case and $\mu=0$ if $f$ is a holomorphic form. 
Proof. We follow closely [1] (see Section 4.3 and Appendix 1). Set $R=4 \pi \sqrt{y}$. Let $H_{s}$ denote either $J_{s}, Y_{s}$ or $K_{s}$. Then $H_{s}$ satisfies the recurrence relations (see (6.1) in [1]),

$$
\frac{\mathrm{d}}{\mathrm{d} x}\left\{(R \sqrt{x})^{s+1} H_{s+1}(R \sqrt{x})\right\}= \pm \frac{R^{2}}{2}(R \sqrt{x})^{s} H_{s}(R \sqrt{x})
$$

where the - appears only for $H_{s}=K_{s}$. By the definition of $\mathcal{V}^{ \pm}(y)$, we only need to prove that

$$
\begin{aligned}
\mathcal{V}(y):= & \int_{0}^{\infty} v(x) H_{s}(R \sqrt{x}) \mathrm{d} x \\
& \ll_{f, A, B, j, \varepsilon} Y(1+R \sqrt{Y})^{-\frac{1}{2}}\left(1+\frac{1}{R \sqrt{Y}}\right)^{2|\operatorname{Im} \mu|+\varepsilon}\left(\frac{1}{R \sqrt{Y}}+\frac{1}{R^{2} Y}\right)^{j} .
\end{aligned}
$$

By integration by parts $j$ times with respect to $x$ and (2.4), we have

$$
\mathcal{V}(y)=\left(\mp \frac{R^{2}}{2}\right)^{-j} \int_{0}^{\infty} \frac{\mathrm{d}^{j}}{\mathrm{~d} x^{j}}\left\{v(x)(R \sqrt{x})^{-s}\right\}(R \sqrt{x})^{s+j} H_{s+j}(R \sqrt{x}) \mathrm{d} x .
$$

Note that

$$
\frac{\mathrm{d}^{j}}{\mathrm{~d} x^{j}}\left\{v(x)(R \sqrt{x})^{-s}\right\}=R^{-s} \sum_{i=0}^{j} C_{j}^{i} v^{(i)}(x)\left(-\frac{s}{2}\right)\left(-\frac{s}{2}-1\right) \cdots\left(-\frac{s}{2}-j+i+1\right) x^{-\frac{s}{2}-(j-i)}
$$

and by Proposition 6.2 in [1], for any $\varepsilon>0$,

$$
H_{s+j}(R \sqrt{x}) \ll_{f, j, \varepsilon}(1+R \sqrt{x})^{-\frac{1}{2}}\left(1+\frac{1}{R \sqrt{x}}\right)^{j+2|\operatorname{Im} \mu|+\varepsilon} .
$$

Plugging these estimates into (2.6) we obtain (2.5). This proves Lemma 2.

The following Voronoi formula for $r_{\ell}(n)$ is due to Luo (see [12, Lemma 2).

Lemma 3. Let a be an integer coprime to $q$, ad $\equiv 1(\bmod q)$ and

$$
\epsilon_{d}= \begin{cases}1, & \text { if } d \equiv 1(\bmod 4) \\ i, & \text { if } d \equiv-1(\bmod 4) .\end{cases}
$$

If $w(x)$ is a compactly supported smooth function on $\mathbb{R}^{+}$, then

$$
\begin{aligned}
\sum_{n \geq 1} r_{\ell}(n) e\left(\frac{a n}{q}\right) w(n)= & \left(\frac{2 \pi i}{q}\right)^{\frac{\ell}{2}} \Gamma\left(\frac{\ell}{2}\right)^{-1}\left(\left(\frac{q}{d}\right) \epsilon_{d}^{-1}\right)^{\ell} \tilde{w}\left(\frac{\ell}{2}\right) \\
& +\frac{2 \pi i^{\frac{\ell}{2}}}{q}\left(\left(\frac{q}{d}\right) \epsilon_{d}^{-1}\right)^{\ell} \sum_{n \geq 1} r_{\ell}(n) e\left(-\frac{d n}{q}\right) n^{\frac{1-\frac{\ell}{2}}{2}} \mathcal{W}\left(\frac{n}{q^{2}}\right)
\end{aligned}
$$

where $\tilde{w}(s)=\int_{0}^{\infty} w(x) x^{s-1} \mathrm{~d} x$ and

$$
\mathcal{W}(y)=\int_{0}^{\infty} w(x) x^{\frac{\ell}{2}-1} J_{\frac{\ell}{2}-1}(4 \pi \sqrt{x y}) d x .
$$

$\mathcal{W}(y)$ has the following properties. 
Lemma 4. If $w(x)$ is a smooth function supported on $[A Y, B Y](B>A>0)$ satisfying $w^{(j)}(x) \ll_{A, B, j}(\Delta / Y)^{j}(\log \Delta \asymp \log Y)$, then for any $s \geq 0$ and any integer $j \geq 0$,

$\mathcal{W}_{s}(y)=\int_{0}^{\infty} w(x) x^{\frac{s}{2}} J_{s}(4 \pi \sqrt{x y}) d x \ll_{s, A, B, j} Y^{1+\frac{s}{2}}(1+\sqrt{y Y})^{-\frac{1}{2}}\left(1+\frac{1}{\sqrt{y Y}}\right)^{-s}\left(\frac{\Delta}{1+\sqrt{y Y}}\right)^{j}$.

Proof. The proof is very similar as that of Lemma 2 and one uses the estimate (see Proposition 6.1 in [1])

$$
J_{s}(R \sqrt{x}) \ll_{s}(1+R \sqrt{x})^{-\frac{1}{2}}\left(1+\frac{1}{R \sqrt{x}}\right)^{-s}
$$

for any $s \geq 0$.

For the properties of Bessel functions, we quote the following lemma (see [3], page 920, 8.451-1, 8.451-2 and 8.451-6).

Lemma 5. For $s \in \mathbb{C}$ and $x \gg 1$, we have

$$
\begin{aligned}
& J_{s}(x)=\sqrt{\frac{2}{\pi x}}\left\{\cos \left(x-\frac{\pi}{2} s-\frac{\pi}{4}\right)-\frac{4 s^{2}-1}{8 x} \sin \left(x-\frac{\pi}{2} s-\frac{\pi}{4}\right)+O_{s}\left(x^{-2}\right)\right\}, \\
& Y_{s}(x)=\sqrt{\frac{2}{\pi x}}\left\{\sin \left(x-\frac{\pi s}{2}-\frac{\pi}{4}\right)+\frac{4 s^{2}-1}{8 x} \cos \left(x-\frac{\pi s}{2}-\frac{\pi}{4}\right)+O_{s}\left(x^{-2}\right)\right\}, \\
& K_{s}(x)=\sqrt{\frac{\pi}{2 x}} e^{-x}\left\{1+O_{s}\left(x^{-1}\right)\right\} .
\end{aligned}
$$

\section{Proof of Theorem 1}

Let $\phi(x)$ be a smooth function which is supported on $[1 / 2,1]$ and identically equal to 1 on $\left[1 / 2+\Delta^{-1}, 1-\Delta^{-1}\right]$ with $\log \Delta \asymp \log X$ to be chosen later, satisfying $\phi^{(j)}(x) \ll_{j} \Delta^{j}$ for all integers $j \geq 0$. Then

$$
\sum_{X / 2<n \leq X} \lambda_{f}(n+h) r_{\ell}(n)=\sum_{n \geq 1} \lambda_{f}(n+h) r_{\ell}(n) \phi\left(\frac{n}{X}\right)+E_{h}(X),
$$

where

$$
E_{h}(X)=\sum_{X / 2<n \leq X} \lambda_{f}(n+h) r_{\ell}(n)\left(1-\phi\left(\frac{n}{X}\right)\right) \ll_{\ell, \varepsilon} X^{\frac{\ell}{2}+\theta+\varepsilon} \Delta^{-1},
$$

using the bound $r_{\ell}(n) \ll_{\ell, \varepsilon} n^{\frac{\ell}{2}-1+\varepsilon}$ and $\lambda_{f}(n) \ll n^{\theta+\varepsilon}$ for any $\varepsilon>0$.

Denote the first term on the right side of $(3.1)$ by $\mathcal{S}_{h}(X)$. Then we have

$$
\mathcal{S}_{h}(X)=\sum_{n \geq 1} \lambda_{f}(n+h) r_{\ell}(n) \phi\left(\frac{n}{X}\right) \varphi\left(\frac{n+h}{X+h}\right),
$$


where $\varphi(x)$ is a smooth function supported on [1/4,5/4] and equals 1 if $x \in[1 / 2,1]$, satisfying $\varphi^{(j)}(x) \ll_{j} 1$. Let $\delta(i, j)=\left\{\begin{array}{ll}1, & \text { if } i=j, \\ 0, & \text { otherwise. }\end{array}\right.$ Then

$$
\delta(i, j)=\int_{0}^{1} e((i-j) \beta) \mathrm{d} \beta .
$$

and

$$
\begin{aligned}
\mathcal{S}_{h}(X) & =\sum_{n \geq 1} r_{\ell}(n) \phi\left(\frac{n}{X}\right) \sum_{m \geq 1} \lambda_{f}(m) \varphi\left(\frac{m}{X+h}\right) \delta(n+h, m) \\
& =\int_{0}^{1} e(h \beta) \sum_{n \geq 1} r_{\ell}(n) \phi\left(\frac{n}{X}\right) e(\beta n) \sum_{m \geq 1} \lambda_{f}(m) \varphi\left(\frac{m}{X+h}\right) e(-\beta m) \mathrm{d} \beta .
\end{aligned}
$$

Applying Jutila's circle method (see [9]), we approximate $\delta(i, j)$ by

$$
\widetilde{\delta}(i, j)=\int_{0}^{1} \widetilde{I}_{\mathcal{Q}, \delta}(\beta) e((i-j) \beta) \mathrm{d} \beta,
$$

and $\mathcal{S}_{h}(X)$ by

$$
\widetilde{\mathcal{S}}_{h}(X)=\int_{0}^{1} \widetilde{I}_{\mathcal{Q}, \delta}(\beta) e(h \beta) \sum_{n \geq 1} r_{\ell}(n) \phi\left(\frac{n}{X}\right) e(\beta n) \sum_{m \geq 1} \lambda_{f}(m) \varphi\left(\frac{m}{X+h}\right) e(-\beta m) \mathrm{d} \beta,
$$

where $\widetilde{I}_{\mathcal{Q}, \delta}(\beta)$ is defined in (1.3), $Q>0$ is a parameter to be chosen soon, $\mathcal{Q} \subset[1, Q]$, $L=\sum_{q \in \mathcal{Q}} \phi(q)$ and $Q^{-2} \leq \delta \leq Q^{-1}$.

By Cauchy's inequality and (1.4), we have, for $L \gg_{\varepsilon} Q^{2-\varepsilon}$,

$$
\begin{aligned}
& \left|\mathcal{S}_{h}(X)-\widetilde{\mathcal{S}}_{h}(X)\right| \\
\leq & \int_{0}^{1}\left|1-\widetilde{I}_{\mathcal{Q}, \delta}(\beta)\right|\left|\sum_{n \geq 1} r_{\ell}(n) \phi\left(\frac{n}{X}\right) e(\beta n)\right|\left|\sum_{m \geq 1} \lambda_{f}(m) \varphi\left(\frac{m}{X+h}\right) e(-\beta m)\right| \mathrm{d} \beta \\
\ll_{f, \varepsilon} \quad & X^{\frac{1}{2}+\varepsilon}\left(\int_{0}^{1}\left|1-\widetilde{I}_{\mathcal{Q}, \delta}(\beta)\right|^{2} \mathrm{~d} \beta\right)^{1 / 2}\left(\int_{0}^{1}\left|\sum_{n \geq 1} r_{\ell}(n) \phi\left(\frac{n}{X}\right) e(\beta n)\right|^{2} \mathrm{~d} \beta\right)^{1 / 2} \\
\ll_{f, \ell, \varepsilon} & \frac{X^{\frac{\ell}{2}+\varepsilon}}{\sqrt{\delta} Q},
\end{aligned}
$$

where we have used the trivial bound $r_{\ell}(n) \ll_{\ell, \varepsilon} n^{\frac{\ell}{2}-1+\varepsilon}$ and the estimate (see (4.2) in [1])

$$
\sum_{n \leq x} \lambda_{f}(n) e(\alpha n) \ll_{f} x^{\frac{1}{2}} \log 2 x
$$

which is uniform in $\alpha \in \mathbb{R}$. Take $\delta=X^{-1}$. We have

$$
\mathcal{S}_{h}(X)=\widetilde{\mathcal{S}}_{h}(X)+O_{f, \ell, \varepsilon}\left(X^{\frac{\ell+1}{2}+\varepsilon} Q^{-1}\right) .
$$


In the following, we estimate $\widetilde{\mathcal{S}}_{h}(X)$ in (3.3). By (1.3), we have

$$
\widetilde{\mathcal{S}}_{h}(X)=\frac{1}{2 \delta} \int_{-\delta}^{\delta} \widetilde{\mathcal{S}}_{h}(X, \beta) e(h \beta) \mathrm{d} \beta,
$$

where

$$
\begin{aligned}
\widetilde{\mathcal{S}}_{h}(X, \beta)=\frac{1}{L} \sum_{q \in \mathcal{Q}} \sum_{\substack{a=1 \\
(a, q)=1}}^{q} e\left(\frac{a h}{q}\right) & \sum_{n \geq 1} r_{\ell}(n) e\left(\frac{a n}{q}\right) \phi\left(\frac{n}{X}\right) e(\beta n) \\
& \times \sum_{m \geq 1} \lambda_{f}(m) e\left(-\frac{a m}{q}\right) \varphi\left(\frac{m}{X+h}\right) e(-\beta m) .
\end{aligned}
$$

Now we choose the set of moduli $\mathcal{Q}$ as follows

$$
\mathcal{Q}=\{4 D p: p \in[Q /(8 D), Q /(4 D)] \text { is prime and }(p, 2 D h)=1\} .
$$

Then the requirement $L \gg_{\varepsilon} Q^{2-\varepsilon}$ is satisfied. Applying Lemma 1 with $v(x)=\varphi\left(\frac{x}{X+h}\right) e(-\beta x)$ to the $m$-sum in (3.6), we have

$$
\begin{aligned}
& \sum_{m \geq 1} \lambda_{f}(m) e\left(-\frac{a m}{q}\right) \varphi\left(\frac{m}{X+b}\right) e(-\beta m) \\
= & \frac{\chi_{D}(-d)}{q} \sum_{m \geq 1} \lambda_{f}(m) e\left(\frac{d m}{q}\right) \mathcal{V}_{\beta}^{+}\left(\frac{m}{q^{2}}\right) \\
& +\omega_{f} \frac{\Gamma\left(\frac{1}{2}+i \mu-\frac{\kappa}{2}\right)}{\Gamma\left(\frac{1}{2}+i \mu+\frac{\kappa}{2}\right)} \frac{\chi_{D}(-d)}{q} \sum_{m \geq 1} \lambda_{f}(m) e\left(-\frac{d m}{q}\right) \mathcal{V}_{\beta}^{-}\left(\frac{m}{q^{2}}\right),
\end{aligned}
$$

where $a d \equiv 1(\bmod q)$ and

$$
\mathcal{V}_{\beta}^{ \pm}(y)=\int_{0}^{\infty} \varphi\left(\frac{x}{X+h}\right) e(-\beta x) \mathcal{H}_{f}^{ \pm}(4 \pi \sqrt{x y}) \mathrm{d} x
$$

with $\mathcal{H}_{f}^{ \pm}(x)$ defined in (2.1)-(2.3). Note that for $|\beta| \leq \delta=X^{-1}, x^{j} v^{(j)}(x) \ll_{j} 1$. By Lemma 2 , the contribution from $m X / q^{2} \gg X^{\varepsilon}$ is negligible. Thus

$$
\widetilde{\mathcal{S}}_{h}(X, \beta)=\widetilde{\mathcal{S}}_{h}(X, \beta,+)+\omega_{f} \frac{\Gamma\left(\frac{1}{2}+i \mu-\frac{\kappa}{2}\right)}{\Gamma\left(\frac{1}{2}+i \mu+\frac{\kappa}{2}\right)} \widetilde{\mathcal{S}}_{h}(X, \beta,-)+O_{\varepsilon}(1),
$$

where

$$
\begin{aligned}
\widetilde{\mathcal{S}}_{h}(X, \beta, \pm)= & \frac{1}{L} \sum_{q \in \mathcal{Q}} \frac{1}{q} \sum_{\substack{a=1 \\
(a, q)=1}}^{q} \chi_{D}(-d) e\left(\frac{a h}{q}\right) \sum_{1 \leq m \ll Q^{2} X^{\varepsilon} / X} \lambda_{f}(m) e\left( \pm \frac{d m}{q}\right) \mathcal{V}_{\beta}^{ \pm}\left(\frac{m}{q^{2}}\right) \\
& \sum_{n \geq 1} r_{\ell}(n) e\left(\frac{a n}{q}\right) \phi\left(\frac{n}{X}\right) e(\beta n) .
\end{aligned}
$$


Applying Lemma 3 with $w(x)=\phi\left(\frac{x}{X}\right) e(\beta x)$ to the $n$-sum in (3.9), we have

$$
\begin{aligned}
\sum_{n \geq 1} r_{\ell}(n) e\left(\frac{a n}{q}\right) \phi\left(\frac{n}{X}\right) e(\beta n)= & \left(\frac{2 \pi i}{q}\right)^{\frac{\ell}{2}} \Gamma\left(\frac{\ell}{2}\right)^{-1}\left(\left(\frac{q}{d}\right) \epsilon_{d}^{-1}\right)^{\ell} \tilde{w}_{\beta}\left(\frac{\ell}{2}\right) \\
& +\frac{2 \pi i^{\frac{\ell}{2}}}{q}\left(\left(\frac{q}{d}\right) \epsilon_{d}^{-1}\right)^{\ell} \sum_{n \geq 1} r_{\ell}(n) e\left(-\frac{d n}{q}\right) n^{\frac{1-\frac{\ell}{2}}{2}} \mathcal{W}_{\beta}\left(\frac{n}{q^{2}}\right)
\end{aligned}
$$

where

$$
\tilde{w}_{\beta}(s)=\int_{0}^{\infty} \phi\left(\frac{x}{X}\right) e(\beta x) x^{s-1} \mathrm{~d} x,
$$

and

$$
\mathcal{W}_{\beta}(y)=\int_{0}^{\infty} \phi\left(\frac{x}{X}\right) e(\beta x) x^{\frac{\frac{\ell}{2}-1}{2}} J_{\frac{\ell}{2}-1}(4 \pi \sqrt{x y}) d x
$$

Note that for $|\beta| \leq \delta=X^{-1}, w^{(j)}(x) \ll_{j}(\Delta / X)^{j}$. By Lemma 4, the contribution from $n X / q^{2} \gg \Delta^{2} X^{\varepsilon}$ in the second sum in (3.10) is negligible.

Plugging (3.10) into (3.9) we obtain

$$
\widetilde{\mathcal{S}}_{h}(X, \beta, \pm)=\widetilde{\mathcal{S}}_{h}^{0}(X, \beta, \pm)+\widetilde{\mathcal{S}}_{h}^{b}(X, \beta, \pm)+O_{\varepsilon}(1),
$$

where

$$
\begin{aligned}
\widetilde{\mathcal{S}}_{h}^{0}(X, \beta, \pm)= & \frac{(2 \pi i)^{\frac{\ell}{2}}}{\Gamma(\ell / 2)} \frac{\tilde{w}_{\beta}(\ell / 2)}{L} \sum_{q \in \mathcal{Q}} \frac{1}{q^{\frac{\ell}{2}+1}} \sum_{1 \leq m \ll Q^{2} X^{\varepsilon} / X} \lambda_{f}(m) \mathcal{V}_{\beta}^{ \pm}\left(\frac{m}{q^{2}}\right) \\
& \times \sum_{\substack{a=1 \\
a d \equiv 1(\bmod q)}} \chi_{D}(-d)\left(\left(\frac{q}{d}\right) \epsilon_{d}^{-1}\right)^{\ell} e\left(\frac{h a \pm m d}{q}\right), \\
\widetilde{\mathcal{S}}_{h}^{b}(X, \beta, \pm)= & \frac{2 \pi i^{\frac{\ell}{2}}}{L} \sum_{q \in \mathcal{Q}} \frac{1}{q^{2}} \sum_{1 \leq m \ll Q^{2} X^{\varepsilon} / X} \lambda_{f}(m) \mathcal{V}_{\beta}^{ \pm}\left(\frac{m}{q^{2}}\right) \sum_{1 \leq n \ll \Delta^{2} Q^{2} X^{\varepsilon} / X} r_{\ell}(n) n^{\frac{1-\frac{\ell}{2}}{2}} \mathcal{W}_{\beta}\left(\frac{n}{q^{2}}\right) \\
& \times \sum_{\substack{a=1 \\
a d \equiv 1(\bmod q)}}^{q} \chi_{D}(-d)\left(\left(\frac{q}{d}\right) \epsilon_{d}^{-1}\right)^{\ell} e\left(\frac{h a+( \pm m-n) d}{q}\right) .
\end{aligned}
$$


Bounding the character sum. (i) $\ell$ is even. In this case $\left(\left(\frac{q}{d}\right) \epsilon_{d}^{-1}\right)^{\ell}=\left(\left(\frac{-q}{a}\right) \epsilon_{a}\right)^{\ell}=$ $\chi_{4}(a)^{\frac{\ell}{2}}$. By our choice of $\mathcal{Q}$ in (3.7), we have, for $M \in \mathbb{Z}$,

$$
\begin{aligned}
& \sum_{\substack{a=1 \\
a d \equiv 1(\bmod q)}}^{q} \chi_{D}(-d)\left(\left(\frac{q}{d}\right) \epsilon_{d}^{-1}\right)^{\ell} e\left(\frac{h a+M d}{q}\right) \\
= & \sum_{\substack{a_{1} \bmod 4 D \\
a_{1} \bar{a}_{1} \equiv(\bmod 4 D)}} \chi_{4}\left(a_{1} p\right)^{\frac{\ell}{2}} \chi_{D}\left(-\bar{a}_{1} \bar{p}\right) e\left(\frac{h a_{1}+\bar{p}^{2} M \bar{a}_{1}}{4 D}\right) \sum_{\substack{a_{2} \bmod p \\
a_{2} \bar{a}_{2} \equiv 1(\bmod p)}} e\left(\frac{h a_{2}+\overline{4 D}^{2} M \bar{a}_{2}}{p}\right) \\
\ll_{D} \sqrt{p} & \sqrt{p}
\end{aligned}
$$

by Weil's bound for Kloosterman sum.

(ii) $\ell$ is odd. In this case $\left(\left(\frac{q}{d}\right) \epsilon_{d}^{-1}\right)^{\ell}=\left(\left(\frac{-q}{a}\right) \epsilon_{a}\right)^{\ell}=\left(\frac{-q}{a}\right) \epsilon_{a}^{\ell}=\left(\frac{q}{a}\right) \chi_{4}(a) \epsilon_{a}^{\ell}$. By our choice of $\mathcal{Q}$ in (3.7) and quadratic reciprocity, we have, for $M \in \mathbb{Z}$,

$$
\begin{aligned}
& \sum_{\substack{a=1 \\
a d \equiv 1(\bmod q)}}^{q} \chi_{D}(-d)\left(\left(\frac{q}{d}\right) \epsilon_{d}^{-1}\right)^{\ell} e\left(\frac{h a+M d}{q}\right) \\
= & \sum_{\substack{a_{1} \bmod 4 D \\
a_{1} \bar{a}_{1} \equiv 1(\bmod 4 D)}}\left(\frac{p a_{1}}{4 D}\right)(-1)^{\frac{p-1}{2} \frac{p a_{1}-1}{2}} \chi_{4}\left(p a_{1}\right) \chi_{D}\left(-\bar{a}_{1} \bar{p}\right) \epsilon_{p a_{1}}^{\ell} e\left(\frac{h a_{1}+\bar{p}^{2} M \bar{a}_{1}}{4 D}\right) \\
& \sum_{\substack{a_{2} \bmod p \\
a_{2} \bar{a}_{2} \equiv 1(\bmod p) \\
\sqrt{p}}}\left(\frac{4 D a_{2}}{p}\right) e\left(\frac{h a_{2}+\overline{4 D}^{2} M \bar{a}_{2}}{p}\right) \\
\ll_{D} &
\end{aligned}
$$

by the well known bound for Salié sums (see Corollary 4.10 in [8]).

Bounding $\widetilde{\mathcal{S}}_{h}^{0}(X, \beta, \pm)$. Denote $\vartheta=|\operatorname{Im} \mu|$. Then by Selberg's bound $\vartheta \leq 1 / 4$. By Lemma 2, for $p \in[Q /(8 D), Q /(4 D)]$, we have

$$
\begin{aligned}
\sum_{1 \leq m \ll Q^{2} X^{\varepsilon} / X} \lambda_{f}(m) \mathcal{V}_{\beta}^{ \pm}\left(\frac{m}{q^{2}}\right) & \ll X \sum_{1 \leq m \ll Q^{2} X^{\varepsilon} / X}\left|\lambda_{f}(m)\right|\left(1+\sqrt{\frac{16 D^{2} p^{2}}{m X}}\right)^{2 \vartheta+\varepsilon} \\
& \ll_{f} X \sum_{1 \leq \frac{m X}{16 D^{2} p^{2}} \ll X^{\varepsilon}}\left|\lambda_{f}(m)\right|+X \sum_{\frac{m X}{16 D^{2} p^{2}} \leq 1}\left|\lambda_{f}(m)\right|\left(\frac{Q^{2}}{m X}\right)^{\vartheta+\varepsilon} \\
& \ll_{f} Q^{2} X^{\varepsilon}
\end{aligned}
$$

by the Rankin-Selberg estimate

$$
\sum_{m \leq x}\left|\lambda_{f}(m)\right|^{2} \ll_{f} x
$$


Recall that $L \gg_{\varepsilon} Q^{2-\varepsilon}$. Bounding the integral $\tilde{w}_{\beta}\left(\frac{\ell}{2}\right)$ in (3.11) trivially and by (3.14)(3.16), we have

$$
\begin{aligned}
\widetilde{\mathcal{S}}_{h}^{0}(X, \beta, \pm) & \ll_{f, \ell, \varepsilon} \frac{X^{\frac{\ell}{2}}}{L} \sum_{p \in[Q /(8 D), Q /(4 D)]} \frac{1}{p^{(\ell+1) / 2}} \sum_{1 \leq m \ll Q^{2} X^{\varepsilon} / X}\left|\lambda_{f}(m)\right|\left|\mathcal{V}^{ \pm}\left(\frac{m}{16 D^{2} p^{2}}\right)\right| \\
& \ll_{f, \ell, \varepsilon} \frac{X^{\frac{\ell}{2}+\varepsilon}}{Q^{\frac{\ell-1}{2}}} .
\end{aligned}
$$

Bounding $\widetilde{\mathcal{S}}_{h}^{b}(X, \beta, \pm)$. First, for $n \ll \Delta^{2} Q^{2} X^{\varepsilon} / X$, we estimate $\mathcal{W}_{\beta}\left(n / q^{2}\right)$ more precisely. By (3.12) we have

$$
\mathcal{W}_{\beta}\left(\frac{n}{q^{2}}\right)=X^{\frac{\ell}{4}+\frac{1}{2}} \int_{0}^{\infty} \phi(x) e(\beta X x) x^{\frac{\ell}{2}-1} J_{\frac{\ell}{2}-1}\left(\frac{4 \pi \sqrt{n X x}}{q}\right) d x .
$$

Set $R=\frac{4 \pi \sqrt{n X}}{q}$. By partial integration once with respect to $x$ and applying the recurrence relation $\left(x^{s} J_{s}(x)\right)^{\prime}=x^{s} J_{s-1}(x)$, we have

$\left.\mathcal{W}_{\beta}\left(\frac{n}{q^{2}}\right)=X^{\frac{\ell}{4}+\frac{1}{2}} R^{-\frac{\ell}{2}+1}\left(\frac{-2}{R^{2}}\right) \int_{0}^{\infty} \frac{d}{d x}\{\phi(x) e(\beta X x)\}(R \sqrt{x})^{\frac{\ell}{2}} J_{\frac{\ell}{2}}(R \sqrt{x}) d x:=\mathcal{E}_{1}+\mathcal{E}_{23} 3.19\right)$

where

$$
\begin{aligned}
& \mathcal{E}_{1}=-4 \pi i \beta X^{\frac{\ell}{4}+\frac{3}{2}} R^{-\frac{\ell}{2}-1} \int_{0}^{\infty} \phi(x) e(\beta X x)(R \sqrt{x})^{\frac{\ell}{2}} J_{\frac{\ell}{2}}(R \sqrt{x}) d x, \\
& \mathcal{E}_{2}=-2 X^{\frac{\ell}{4}+\frac{1}{2}} R^{-\frac{\ell}{2}-1} \int_{0}^{\infty} \phi^{\prime}(x) e(\beta X x)(R \sqrt{x})^{\frac{\ell}{2}} J_{\frac{\ell}{2}}(R \sqrt{x}) d x .
\end{aligned}
$$

Using the bound $J_{s}(x) \ll \min \left\{x^{s}, x^{-\frac{1}{2}}\right\}$ for $s \geq 0$, we have, for $|\beta| \leq X^{-1}$,

$$
\mathcal{E}_{1} \ll X^{\frac{\ell}{4}+\frac{1}{2}} R^{-1} \min \left\{R^{\frac{\ell}{2}}, R^{-1 / 2}\right\}
$$

and

$$
\begin{aligned}
\mathcal{E}_{2} & =-2 X^{\frac{\ell}{4}+\frac{1}{2}} R^{-\frac{\ell}{2}-1}\left\{\int_{1}^{1+2 \Delta^{-1}}+\int_{2-2 \Delta^{-1}}^{2}\right\} \phi^{\prime}(x) e(\beta X x)(R \sqrt{x})^{\frac{\ell}{2}} J_{\frac{\ell}{2}}(R \sqrt{x}) d x \\
& \ll X^{\frac{\ell}{4}+\frac{1}{2}} R^{-1} \min \left\{R^{\frac{\ell}{2}}, R^{-1 / 2}\right\} .
\end{aligned}
$$

By (3.19)-(3.21), we have

$$
\mathcal{W}_{\beta}\left(\frac{n}{q^{2}}\right) \ll X^{\frac{\ell}{4}+\frac{1}{2}} R^{-1} \min \left\{R^{\frac{\ell}{2}}, R^{-1 / 2}\right\} \ll X^{\frac{\ell}{4}+\frac{1}{2}} \min \left\{\left(\frac{\sqrt{n X}}{q}\right)^{\frac{\ell}{2}-1},\left(\frac{q}{\sqrt{n X}}\right)^{\frac{3}{2}}\right\}(
$$


By (3.16), (3.22) and the estimate $r_{\ell}(n) \ll n^{\frac{\ell}{2}-1+\varepsilon}$, we have

$$
\begin{aligned}
\widetilde{\mathcal{S}}_{h}^{b}(X, \beta, \pm) & \ll \sum_{p \in[Q /(8 D), Q /(4 D)]} \frac{1}{p^{3 / 2}} \sum_{n \ll \Delta^{2} Q^{2} X^{\varepsilon} / X} n^{\frac{\ell}{4}-\frac{1}{2}} X^{\frac{\ell}{4}+\frac{1}{2}} \min \left\{\left(\frac{\sqrt{n X}}{p}\right)^{\frac{\ell}{2}-1},\left(\frac{p}{\sqrt{n X}}\right)^{\frac{3}{2}}\right\} \\
& \ll \frac{X^{\frac{\ell}{4}+\frac{1}{2}+\varepsilon}}{\sqrt{Q}} \sum_{n \ll \Delta^{2} Q^{2} X^{\varepsilon} / X} n^{\frac{\ell}{4}-\frac{1}{2}} \min \left\{\left(\frac{\sqrt{n X}}{Q}\right)^{\frac{\ell}{2}-1},\left(\frac{Q}{\sqrt{n X}}\right)^{\frac{3}{2}}\right\} \\
& \ll \frac{X^{\frac{\ell}{4}+\frac{1}{2}+\varepsilon}}{\sqrt{Q}}\left\{\sum_{Q^{2} / X<n \ll Q^{2} \Delta^{2} X^{\varepsilon} / X}\left(\frac{Q}{\sqrt{n X}}\right)^{\frac{3}{2}}+\sum_{n \leq Q^{2} / X}\left(\frac{\sqrt{n X}}{Q}\right)^{\frac{\ell}{2}-1}\right\} \\
& \ll X^{\frac{\ell}{4}+\frac{1}{2}+\varepsilon} Q^{-\frac{1}{2}}+X^{\frac{\ell}{4}-\frac{1}{2}+\varepsilon} Q^{\frac{3}{2}} \Delta^{\frac{1}{2}} .
\end{aligned}
$$

By (3.4), (3.5), (3.8), (3.13), (3.18) and (3.23), we have, for $Q<X$,

$$
\mathcal{S}_{h}(X) \ll \frac{X^{\frac{\ell}{2}+\varepsilon}}{Q^{\frac{\ell-1}{2}}}+X^{\frac{\ell}{4}-\frac{1}{2}+\varepsilon} Q^{\frac{3}{2}} \Delta^{\frac{1}{2}}+X^{\frac{\ell+1}{2}+\varepsilon} Q^{-1} .
$$

Note that the first term is smaller that the third term for $\ell \geq 2$ and $Q<X$. Take $Q=X^{\frac{\ell+4}{10}} \Delta^{-\frac{1}{5}}$. Then

$$
\mathcal{S}_{h}(X) \ll_{f, \ell, \varepsilon} X^{\frac{4 \ell+1}{10}+\varepsilon} \Delta^{\frac{1}{5}}
$$

By (3.1), (3.2) and (3.25),

$$
\sum_{X / 2<n \leq X} \lambda_{f}(n+h) r_{\ell}(n) \ll_{f, \ell, \varepsilon} X^{\frac{\ell}{2}+\theta+\varepsilon} \Delta^{-1}+X^{\frac{4 \ell+1}{10}+\varepsilon} \Delta^{\frac{1}{5}} .
$$

By taking $\Delta=X^{\frac{\ell-1}{12}+\frac{5 \theta}{6}}$ in (3.26) we obtain

$$
\sum_{X / 2<n \leq X} \lambda_{f}(n+h) r_{\ell}(n) \ll_{f, \ell, \varepsilon} X^{\frac{\ell}{2}-\frac{\ell-1-2 \theta}{12}+\varepsilon} .
$$

This proves Theorem 1 .

\section{Proof of Theorem 2}

Denote the sum in Theorem 2 by $\mathcal{S}_{h}^{*}(X)$, By the Hardy-Littlewood-Kloosterman circle method (see for example, [8], Section 11.4), we have

$$
\mathcal{S}_{h}^{*}(X)=\int_{0}^{1} \mathscr{F}^{2}(\alpha) \mathscr{G}(\alpha) \mathrm{d} \alpha,
$$

where

$$
\mathscr{F}(\alpha)=\sum_{\substack{|m| \leq \sqrt{X} \\ 12}} e\left(\alpha m^{2}\right)
$$


and

$$
\mathscr{G}(\alpha)=\sum_{n \geq 1} \lambda_{f}(n+h) e(-\alpha n) \phi\left(\frac{n}{X}\right) .
$$

Note that $\mathscr{F}^{2}(\alpha) \mathscr{G}(\alpha)$ is a periodic function of period 1 . We have

$$
\mathcal{S}_{h}^{*}(X)=\int_{-1 /(Q+1)}^{1-1 /(Q+1)} \mathscr{F}^{2}(\alpha) \mathscr{G}(\alpha) \mathrm{d} \alpha,
$$

where $Q=[5 \sqrt{X}]$. Dissecting the unit interval with Farey's points of order $Q$, we have

$$
\mathcal{S}_{h}^{*}(X)=\sum_{q \leq Q} \sum_{a=1}^{q} \int_{\mathcal{M}(a, q)} \mathscr{F}^{2}\left(\frac{a}{q}+\beta\right) \mathscr{G}\left(\frac{a}{q}+\beta\right) \mathrm{d} \beta,
$$

where the $*$ denotes that the sum is restricted by the condition $(a, q)=1, \mathcal{M}(a, q)=$ $\left[-\frac{1}{q\left(q+q^{\prime}\right)}, \frac{1}{q\left(q+q^{\prime \prime}\right)}\right]$, and $\frac{a^{\prime}}{q^{\prime}}, \frac{a}{q}$ and $\frac{a^{\prime \prime}}{q^{\prime \prime}}$ are consecutive Farey fractions which are determined by the conditions

$$
Q<q+q^{\prime}, q+q^{\prime \prime} \leq q+Q, \quad a q^{\prime} \equiv 1(\bmod q), \quad a q^{\prime \prime} \equiv-1(\bmod q) .
$$

Exchanging the order of the summation over $a$ and the integration over $\beta$ as in HeathBrown [5] (see Lemma 7), we have

$$
\mathcal{S}_{h}^{*}(X)=\sum_{q \leq Q_{|\beta| \leq \frac{1}{q Q}}} \sum_{v \bmod q} \varrho(v, q, \beta) \sum_{\substack{a=1 \\(a, q)=1}}^{q} e\left(-\frac{\bar{a} v}{q}\right) \mathscr{F}^{2}\left(\frac{a}{q}+\beta\right) \mathscr{G}\left(\frac{a}{q}+\beta\right) \mathrm{d} \beta(4,
$$

where $\varrho(v, q, \beta)$ satisfies

$$
\varrho(v, q, \beta) \ll \frac{1}{1+|v|}
$$

Here the implied constant is absolute.

For an asymptotic formula for $\mathscr{F}\left(\frac{a}{q}+\beta\right)$, we quote the following result (see [18], Theorem 4.1).

Lemma 6. Suppose $(a, q)=1, q \leq Q$ and $|\beta| \leq \frac{1}{q Q}$. Then we have

$$
\mathscr{F}\left(\frac{a}{q}+\beta\right)=\frac{2 G(a, 0 ; q)}{q} \Phi_{0}(\beta)+\sum_{-\frac{3 q}{2}<b \leq \frac{3 q}{2}} G(a, b ; q) \Phi(b, q, \beta),
$$

where $G(a, b ; q)$ is the Gauss sum

$$
G(a, b ; q)=\sum_{x \bmod q} e\left(\frac{a x^{2}+b x}{q}\right)
$$


$\Phi_{0}(\beta)$ is the integral

$$
\Phi_{0}(\beta)=\int_{0}^{\sqrt{X}} e\left(\beta x^{2}\right) \mathrm{d} x
$$

and $\Phi(b, q, \beta)$ satisfies

$$
\sum_{-\frac{3 q}{2}<b \leq \frac{3 q}{2}}|\Phi(b, q, \beta)| \ll \log (q+2) .
$$

By (4.1) and Lemma 1, we have

$$
\begin{aligned}
\mathscr{G}\left(\frac{a}{q}+\beta\right)= & e\left(\frac{h a}{q}+h \beta\right) \sum_{m \geq 1} \lambda_{f}(m) e\left(-\frac{a m}{q}\right) \phi\left(\frac{m-h}{X}\right) e(-\beta m) \\
= & e\left(\frac{h a}{q}+h \beta\right) \frac{1}{q} \sum_{m \geq 1} \lambda_{f}(m) e\left(\frac{\bar{a} m}{q}\right) \mathcal{V}_{\beta}^{+}\left(\frac{m}{q^{2}}\right) \\
& +\omega_{f}^{*} e\left(\frac{h a}{q}+h \beta\right) \frac{1}{q} \sum_{m \geq 1} \lambda_{f}(m) e\left(-\frac{\bar{a} m}{q}\right) \mathcal{V}_{\beta}^{-}\left(\frac{m}{q^{2}}\right)
\end{aligned}
$$

where $\omega_{f}^{*}$ is a constant depending only on $f$ and

$$
\mathcal{V}_{\beta}^{ \pm}(y)=\int_{0}^{\infty} \phi\left(\frac{x-h}{X}\right) e(-\beta x) \mathcal{H}_{f}^{ \pm}(4 \pi \sqrt{x y}) \mathrm{d} x
$$

with $\mathcal{H}_{f}^{ \pm}(x)$ defined in (2.1)-(2.3). Denote

$$
\mathscr{G}^{ \pm}\left(\frac{a}{q}+\beta\right)=e\left(\frac{h a}{q}+h \beta\right) \frac{1}{q} \sum_{m \geq 1} \lambda_{f}(m) e\left( \pm \frac{\bar{a} m}{q}\right) \mathcal{V}_{\beta}^{ \pm}\left(\frac{m}{q^{2}}\right)
$$

Then by (4.4) and (4.10), we have

$$
\sum_{a=1}^{q} e\left(-\frac{\bar{a} v}{q}\right) \mathscr{F}^{2}\left(\frac{a}{q}+\beta\right) \mathscr{G}^{ \pm}\left(\frac{a}{q}+\beta\right)=\sum_{j=1}^{3} \mathscr{D}_{j}^{ \pm}(v, q, \beta),
$$

where

$$
\begin{aligned}
& \mathscr{D}_{1}^{ \pm}(v, q, \beta)=\frac{4 e(h \beta) \Phi_{0}(\beta)^{2}}{q^{3}} \sum_{m \geq 1} \lambda_{f}(m) \mathcal{V}_{\beta}^{ \pm}\left(\frac{m}{q^{2}}\right) \mathscr{C}(0,0, h, \pm m-v ; q) \\
& \mathscr{D}_{2}^{ \pm}(v, q, \beta)=\frac{4 e(h \beta) \Phi_{0}(\beta)}{q^{2}} \sum_{m \geq 1} \lambda_{f}(m) \mathcal{V}_{\beta}^{ \pm}\left(\frac{m}{q^{2}}\right) \sum_{-\frac{3 q}{2}<b \leq \frac{3 q}{2}} \Phi(b, q, \beta) \mathscr{C}(0, b, h, \pm m-v ; q), \\
& \mathscr{D}_{3}^{ \pm}(v, q, \beta)=\frac{e(h \beta)}{q} \sum_{m \geq 1} \lambda_{f}(m) \mathcal{V}_{\beta}^{ \pm}\left(\frac{m}{q^{2}}\right) \sum_{\substack{-\frac{3 q}{2}<b_{1} \leq \frac{3 q}{2} \\
-\frac{3 q}{2}<b_{2} \leq \frac{3 q}{2} \\
14}} \Phi\left(b_{1}, q, \beta\right) \Phi\left(b_{2}, q, \beta\right) \mathscr{C}\left(b_{1}, b_{2}, h, \pm m-v ; q\right)
\end{aligned}
$$


with

$$
\mathscr{C}\left(b_{1}, b_{2}, h, u ; q\right)=\sum_{a=1}^{q} G\left(a, b_{1} ; q\right) G\left(a, b_{2} ; q\right) e\left(\frac{a h+\bar{a} u}{q}\right), \quad u \in \mathbb{Z} .
$$

The following proposition will be proved in the next section.

Proposition 1. We have

$$
\sum_{m \geq 1}\left|\lambda_{f}(m)\right|\left|\mathcal{V}_{\beta}^{ \pm}\left(\frac{m}{q^{2}}\right)\right| \ll_{f} q^{2}(1+|\beta| X)+\Delta q^{\frac{5}{2}} X^{-\frac{1}{4}}
$$

For the exponential sum $\mathscr{C}\left(b_{1}, b_{2}, h, u ; q\right)$, we have the following estimate.

Proposition 2. Let $q=q_{1} q_{2},\left(2 q_{1}, q_{2}\right)=1,4 q_{1}$ squarefull and $q_{2}$ squarefree. We have

$$
\mathscr{C}\left(b_{1}, b_{2}, h, u ; q\right) \ll q_{1}^{2} q_{2}^{\frac{3}{2}}\left(h, q_{2}\right)^{\frac{1}{2}} .
$$

Proof. Let $q=q_{1} q_{2}$, where $\left(2 q_{1}, q_{2}\right)=1,4 q_{1}$ squarefull, $q_{2}$ square-free. Then we have

$$
\mathscr{C}\left(b_{1}, b_{2}, h, u ; q\right)=\mathscr{C}\left(b_{1}, b_{2}, \bar{q}_{2}^{2} h, u ; q_{1}\right) \mathscr{C}\left(b_{1}, b_{2}, \bar{q}_{1}^{2} h, u ; q_{2}\right) .
$$

By Lemma 5.4 .5 in [7], we have $G(a, b ; q) \ll \sqrt{q}$. Thus

$$
\mathscr{C}\left(b_{1}, b_{2}, \bar{q}_{2}^{2} h, u ; q_{1}\right) \ll q_{1}^{2} .
$$

To estimate $\mathscr{C}\left(b_{1}, b_{2}, \bar{q}_{1}^{2} h, u ; q_{2}\right)$, we factor $q_{2}$ as $q_{2}=p_{1} p_{2} \cdots p_{s}, p_{i}$ prime. Then

$$
\mathscr{C}\left(b_{1}, b_{2}, \bar{q}_{1}^{2} h, u ; q_{2}\right)=\prod_{i=1}^{s} \mathscr{C}\left(b_{1}, b_{2},{\overline{p_{i}^{\prime}}}^{2} \bar{q}_{1}^{2} h, u ; p_{i}\right),
$$

where $p_{i}^{\prime}=q_{2} / p_{i}$. Thus we consider the exponential sum

$$
\mathscr{C}\left(b_{1}, b_{2}, r h, u ; p\right)=\sum_{a=1}^{p} G\left(a, b_{1} ; p\right) G\left(a, b_{2} ; p\right) e\left(\frac{a r h+\bar{a} u}{p}\right), \quad(p, 2 r)=1, \quad p \text { prime. }
$$

By Lemma 5.4.5 in [7], we have, for $(2 a, q)=1$,

$$
G(a, b ; q)=e\left(-\frac{\overline{4} \bar{a} b^{2}}{q}\right) G(a, 0 ; q)=e\left(-\frac{\overline{4} \bar{a} b^{2}}{q}\right)\left(\frac{a}{q}\right) \epsilon_{q} \sqrt{q},
$$

where $\epsilon_{q}$ is defined in (2.7). Thus by Weil's bound for Kloosterman sum, we have

$$
\begin{aligned}
\mathscr{C}\left(b_{1}, b_{2}, r h, u ; p\right) & =\sum_{a=1}^{p}{ }^{*} e\left(-\frac{\overline{4} \bar{a} b_{1}^{2}}{p}\right)\left(\frac{a}{p}\right) \epsilon_{p} \sqrt{p} e\left(-\frac{\overline{4} \bar{a} b_{2}^{2}}{p}\right)\left(\frac{a}{p}\right) \epsilon_{p} \sqrt{p} e\left(\frac{a r h+\bar{a} u}{p}\right) \\
& =p \epsilon_{p}^{2} \sum_{a=1}^{*} e\left(\frac{a r h+\bar{a}\left(u-\overline{4} b_{1}^{2}-\overline{4} b_{2}^{2}\right)}{p}\right) \\
& \ll p^{\frac{3}{2}}\left(r h, u-\overline{4} b_{1}^{2}-\overline{4} b_{2}^{2}, p\right)^{\frac{1}{2}} \\
& \ll p^{\frac{3}{2}}(h, p)^{\frac{1}{2}} .
\end{aligned}
$$


It follows that

$$
\mathscr{C}\left(b_{1}, b_{2}, \bar{q}_{1}^{2} h, u ; q_{2}\right) \ll q_{2}^{\frac{3}{2}}\left(h, q_{2}\right)^{\frac{1}{2}} .
$$

By (4.12)-(4.14), Proposition 2 follows.

By the second derivative test for exponential integrals and the trivial estimate, $\Phi_{0}(\beta)$ in (4.6) is bounded by

$$
\Phi_{0}(\beta) \ll\left(\frac{X}{1+|\beta| X}\right)^{\frac{1}{2}} .
$$

By (4.3), (4.15) and Propositions 1-2, we have, for $q=q_{1} q_{2},\left(2 q_{1}, q_{2}\right)=1,4 q_{1}$ squarefull and $q_{2}$ square-free,

$$
\begin{gathered}
\int_{|\beta| \leq \frac{1}{q Q}} \sum_{v \bmod q} \varrho(v, q, \beta) \mathscr{D}_{1}^{ \pm}(v, q, \beta) \mathrm{d} \beta \\
\ll_{f} \quad(\log Q) \frac{1}{q_{1}^{3} q_{2}^{3}} \int_{|\beta| \leq \frac{1}{q_{1} q_{2} Q}} \frac{X}{1+|\beta| X}\left(q_{1}^{2} q_{2}^{2}(1+|\beta| X)+\Delta q_{1}^{\frac{5}{2}} q_{2}^{\frac{5}{2}} X^{-\frac{1}{4}}\right) q_{1}^{2} q_{2}^{\frac{3}{2}}\left(h, q_{2}\right)^{\frac{1}{2}} \mathrm{~d} \beta \\
\ll_{f} \quad(\log X)^{2}\left(\frac{X q_{1}^{2} q_{2}^{2}}{q_{1} q_{2} Q}+\Delta q_{1}^{\frac{5}{2}} q_{2}^{\frac{5}{2}} X^{-\frac{1}{4}}\right) q_{1}^{-1} q_{2}^{-\frac{3}{2}}\left(h, q_{2}\right)^{\frac{1}{2}},
\end{gathered}
$$

and

$$
\begin{aligned}
& \sum_{q \leq Q} \int_{|\beta| \leq \frac{1}{q Q}} \sum_{v \bmod q} \varrho(v, q, \beta) \mathscr{D}_{1}^{ \pm}(v, q, \beta) \mathrm{d} \beta \\
& \ll_{f, \varepsilon} \frac{X^{1+\varepsilon}}{Q} \sum_{q_{2} \leq Q}\left(h, q_{2}\right)^{\frac{1}{2}} q_{2}^{-\frac{1}{2}} \sum_{\substack{q_{1} \leq Q / q_{2} \\
4 q_{1} \text { squarefull }}} 1+\Delta X^{-\frac{1}{4}+\varepsilon} \sum_{q_{2} \leq Q}\left(h, q_{2}\right)^{\frac{1}{2}} q_{2} \sum_{\substack{q_{1} \leq Q / q_{2} \\
\text { aq }} \text { squarefull }} q_{1}^{\frac{3}{2}} \\
& \ll_{f, \varepsilon} \frac{X^{1+\varepsilon}}{Q} \sum_{q_{2} \leq Q}\left(h, q_{2}\right)^{\frac{1}{2}} q_{2}^{-\frac{1}{2}}\left(\frac{Q}{q_{2}}\right)^{\frac{1}{2}}+\Delta X^{-\frac{1}{4}+\varepsilon} \sum_{q_{2} \leq Q}\left(h, q_{2}\right)^{\frac{1}{2}} q_{2}\left(\frac{Q}{q_{2}}\right)^{2} \\
& \ll_{f, \varepsilon} \frac{X^{1+\varepsilon}}{\sqrt{Q}} \sum_{d_{1} \mid h} \frac{1}{\sqrt{d_{1}}} \sum_{d_{2} \leq Q / d_{1}} \frac{1}{d_{2}}+\Delta X^{-\frac{1}{4}+\varepsilon} Q^{2} \sum_{d_{1} \mid h} \frac{1}{\sqrt{d_{1}}} \sum_{d_{2} \leq Q / d_{1}} \frac{1}{d_{2}} \\
& \ll_{f, \varepsilon} \frac{h^{\varepsilon} X^{1+\varepsilon}}{\sqrt{Q}}+h^{\varepsilon} \Delta X^{-\frac{1}{4}+\varepsilon} Q^{2} \\
& \ll_{f, \varepsilon} \quad \Delta X^{\frac{3}{4}+\varepsilon},
\end{aligned}
$$

uniformly for $1 \leq h \leq X$. 
Similarly, by (4.3), (4.7), (4.15) and Propositions 1-2, we have

$$
\begin{aligned}
& \int_{|\beta| \leq \frac{1}{q Q}} \sum_{v \bmod q} \varrho(v, q, \beta) \mathscr{D}_{2}^{ \pm}(v, q, \beta) \mathrm{d} \beta \\
\ll_{f} & \frac{(\log Q)^{2}}{q_{1}^{2} q_{2}^{2}} \int_{|\beta| \leq \frac{1}{q_{1} q_{2} Q}}\left(\frac{X}{1+|\beta| X}\right)^{\frac{1}{2}}\left(q_{1}^{2} q_{2}^{2}(1+|\beta| X)+\Delta q_{1}^{\frac{5}{2}} q_{2}^{\frac{5}{2}} X^{-\frac{1}{4}}\right) q_{1}^{2} q_{2}^{\frac{3}{2}}\left(h, q_{2}\right)^{\frac{1}{2}} \mathrm{~d} \beta \\
\ll_{f} & (\log X)^{2}\left(\frac{X^{\frac{1}{2}} q_{1}^{2} q_{2}^{2}}{q_{1} q_{2} Q}+\frac{X q_{1}^{2} q_{2}^{2}}{\left(q_{1} q_{2} Q\right)^{\frac{3}{2}}}+\frac{\Delta q_{1}^{\frac{5}{2}} q_{2}^{\frac{5}{2}} X^{-\frac{1}{4}}}{\sqrt{q_{1} q_{2} Q}}\right) q_{2}^{-\frac{1}{2}}\left(h, q_{2}\right)^{\frac{1}{2}}
\end{aligned}
$$

and

$$
\begin{aligned}
& \sum_{q \leq Q_{|\beta| \leq \frac{1}{q Q}}} \sum_{v \bmod q} \varrho(v, q, \beta) \mathscr{D}_{2}^{ \pm}(v, q, \beta) \mathrm{d} \beta \\
\ll_{f, \varepsilon} & \frac{X^{\frac{1}{2}+\varepsilon}}{Q} \sum_{q_{2} \leq Q}\left(h, q_{2}\right)^{\frac{1}{2}} q_{2}^{\frac{1}{2}} \sum_{\substack{q_{1} \leq Q / q_{2} \\
4 q_{1} \text { squarefull }}} q_{1}+\frac{X^{1+\varepsilon}}{Q^{\frac{3}{2}}} \sum_{q_{2} \leq Q}\left(h, q_{2}\right)^{\frac{1}{2}} \sum_{\substack{q_{1} \leq Q / q_{2} \\
4 q_{1} \text { squarefull }}} q_{1}^{\frac{1}{2}} \\
& +\Delta X^{-\frac{1}{4}+\varepsilon} Q^{-\frac{1}{2}} \sum_{q_{2} \leq Q}\left(h, q_{2}\right)^{\frac{1}{2}} q_{2}^{\frac{3}{2}} \sum_{\substack{q_{1} \leq Q / q_{2} \\
4 q_{1} q_{1} \text { quarefull }}} q_{1}^{2} \\
\ll_{f, \varepsilon} & \frac{X^{\frac{1}{2}+\varepsilon}}{Q} \sum_{q_{2} \leq Q}\left(h, q_{2}\right)^{\frac{1}{2}} q_{2}^{\frac{1}{2}}\left(\frac{Q}{q_{2}}\right)^{\frac{3}{2}}+\frac{X^{1+\varepsilon}}{Q^{\frac{3}{2}}} \sum_{q_{2} \leq Q}\left(h, q_{2}\right)^{\frac{1}{2}}\left(\frac{Q}{q_{2}}\right) \\
& +\Delta X^{-\frac{1}{4}+\varepsilon} Q^{-\frac{1}{2}} \sum_{q_{2} \leq Q}\left(h, q_{2}\right)^{\frac{1}{2}} q_{2}^{\frac{3}{2}}\left(\frac{Q}{q_{2}}\right)^{\frac{5}{2}} \\
\ll_{f, \varepsilon} \quad & X^{\frac{1}{2}+\varepsilon} Q^{\frac{1}{2}} \sum_{d_{1} \mid h} d_{1}^{-\frac{1}{2}} \sum_{d_{2} \leq Q / d_{1}} \frac{1}{d_{2}}+\frac{X^{1+\varepsilon}}{Q^{\frac{1}{2}}} \sum_{d_{1} \mid h} d_{1}^{-\frac{1}{2}} \sum_{d_{2} \leq Q / d_{1}} \frac{1}{d_{2}} \\
& +\Delta X^{-\frac{1}{4}+\varepsilon} Q^{2} \sum_{d_{1} \mid h} \frac{1}{\sqrt{d_{1}}} \sum_{d_{2} \leq Q / d_{1}} \frac{1}{d_{2}} \\
\ll_{f, \varepsilon} & h^{\varepsilon} X^{\frac{1}{2}+\varepsilon} Q^{\frac{1}{2}}+\frac{h^{\varepsilon} X^{1+\varepsilon}}{Q^{\frac{1}{2}}}+h^{\varepsilon} \Delta X^{-\frac{1}{4}+\varepsilon} Q^{2} \\
\ll_{f, \varepsilon} & \Delta X^{\frac{3}{4}+\varepsilon}, \quad
\end{aligned}
$$

uniformly for $1 \leq h \leq X$. 
Further, by (4.3), (4.7), (4.15) and Propositions 1-2, we have

$$
\begin{gathered}
\int_{|\beta| \leq \frac{1}{q Q}} \sum_{v \bmod q} \varrho(v, q, \beta) \mathscr{D}_{3}^{ \pm}(v, q, \beta) \mathrm{d} \beta \\
\ll_{f} \frac{(\log Q)^{3}}{q_{1} q_{2}} \int_{|\beta| \leq \frac{1}{q_{1} q_{2} Q}}\left(q_{1}^{2} q_{2}^{2}(1+|\beta| X)+\Delta q_{1}^{\frac{5}{2}} q_{2}^{\frac{5}{2}} X^{-\frac{1}{4}}\right) q_{1}^{2} q_{2}^{\frac{3}{2}}\left(h, q_{2}\right)^{\frac{1}{2}} \mathrm{~d} \beta \\
\ll_{f}(\log X)^{3}\left(\frac{q_{1}^{2} q_{2}^{2}}{q_{1} q_{2} Q}+\frac{X q_{1}^{2} q_{2}^{2}}{\left(q_{1} q_{2} Q\right)^{2}}+\frac{\Delta q_{1}^{\frac{5}{2}} q_{2}^{\frac{5}{2}} X^{-\frac{1}{4}}}{q_{1} q_{2} Q}\right) q_{1} q_{2}^{\frac{1}{2}}\left(h, q_{2}\right)^{\frac{1}{2}}
\end{gathered}
$$

and

$$
\begin{aligned}
& \sum_{q \leq Q_{|\beta| \leq \frac{1}{q Q}}} \sum_{v \bmod q} \varrho(v, q, \beta) \mathscr{D}_{3}^{ \pm}(v, q, \beta) \mathrm{d} \beta \\
& \ll_{f, \varepsilon} \frac{X^{\varepsilon}}{Q} \sum_{q_{2} \leq Q}\left(h, q_{2}\right)^{\frac{1}{2}} q_{2}^{\frac{3}{2}} \sum_{\substack{q_{1} \leq Q / q_{2} \\
4 q_{1} \text { squarefull }}} q_{1}^{2}+\frac{X^{1+\varepsilon}}{Q^{2}} \sum_{q_{2} \leq Q}\left(h, q_{2}\right)^{\frac{1}{2}} q_{2}^{\frac{1}{2}} \sum_{\substack{q_{1} \leq Q / q_{2} \\
4 q_{1} \text { squarefull }}} q_{1} \\
& +\Delta X^{-\frac{1}{4}+\varepsilon} Q^{-1} \sum_{q_{2} \leq Q}\left(h, q_{2}\right)^{\frac{1}{2}} q_{2}^{2} \sum_{\substack{q_{1} \leq Q / q_{2} \\
4 q_{1} \text { squarefull }}} q_{1}^{\frac{5}{2}} \\
& \ll_{f, \varepsilon} \frac{X^{\varepsilon}}{Q} \sum_{q_{2} \leq Q}\left(h, q_{2}\right)^{\frac{1}{2}} q_{2}^{\frac{3}{2}}\left(\frac{Q}{q_{2}}\right)^{\frac{5}{2}}+\frac{X^{1+\varepsilon}}{Q^{2}} \sum_{q_{2} \leq Q}\left(h, q_{2}\right)^{\frac{1}{2}} q_{2}^{\frac{1}{2}}\left(\frac{Q}{q_{2}}\right)^{\frac{3}{2}} \\
& +\Delta X^{-\frac{1}{4}+\varepsilon} Q^{-1} \sum_{q_{2} \leq Q}\left(h, q_{2}\right)^{\frac{1}{2}} q_{2}^{2}\left(\frac{Q}{q_{2}}\right)^{3} \\
& \ll_{f, \varepsilon} \quad X^{\varepsilon} Q^{\frac{3}{2}} \sum_{d_{1} \mid h} d_{1}^{-\frac{1}{2}} \sum_{d_{2} \leq Q / d_{1}} \frac{1}{d_{2}}+\frac{X^{1+\varepsilon}}{Q^{\frac{1}{2}}} \sum_{d_{1} \mid h} d_{1}^{-\frac{1}{2}} \sum_{d_{2} \leq Q / d_{1}} \frac{1}{d_{2}}+\Delta X^{-\frac{1}{4}+\varepsilon} Q^{2} \sum_{d_{1} \mid h} \frac{1}{\sqrt{d_{1}}} \sum_{d_{2} \leq Q / d_{1}} \frac{1}{d_{2}} \\
& \ll_{f, \varepsilon} \quad h^{\varepsilon} X^{\varepsilon} Q^{\frac{3}{2}}+\frac{h^{\varepsilon} X^{1+\varepsilon}}{Q^{\frac{1}{2}}}+h^{\varepsilon} \Delta X^{-\frac{1}{4}+\varepsilon} Q^{2} \\
& \ll_{f, \varepsilon} \Delta X^{\frac{3}{4}+\varepsilon},
\end{aligned}
$$

uniformly for $1 \leq h \leq X$. By (4.2), (4.8), (4.11) and (4.16)-(4.18), Theorem 2 follows.

\section{Proof of Proposition 1}

Recall $\mathcal{V}_{\beta}^{ \pm}(y)$ in (4.9) which we relabel as

$$
\mathcal{V}_{\beta}^{ \pm}(y)=\int_{0}^{\infty} \phi\left(\frac{x-h}{X}\right) e(-\beta x) \mathcal{H}_{f}^{ \pm}(4 \pi \sqrt{x y}) \mathrm{d} x,
$$


where $\mathcal{H}_{f}^{ \pm}(x)$ defined in (2.1)-(2.3). Note that for $y=m / q^{2}, q \leq Q=[5 \sqrt{X}]$ and $x \gg X$, we have $4 \pi \sqrt{x y}>1$. By Lemma 5 , we have

$$
\mathcal{H}_{f}^{-}(4 \pi \sqrt{x y}) \ll_{f} x^{-\frac{5}{4}} y^{-\frac{5}{4}},
$$

and

$$
\mathcal{H}_{f}^{+}(4 \pi \sqrt{x y})=(x y)^{-\frac{1}{4}} \sum_{ \pm} c_{1}^{ \pm} e( \pm 2 \sqrt{x y})+(x y)^{-\frac{3}{4}} \sum_{ \pm} c_{2}^{ \pm} e( \pm 2 \sqrt{x y})+O_{f}\left(x^{-\frac{5}{4}} y^{-\frac{5}{4}}\right)
$$

where $c_{1}^{ \pm}$and $c_{2}^{ \pm}$are absolute constants depending only on the weight $\kappa$ or the spectral parameter $\mu$. Plugging (5.2) and (5.3) into (5.1), we have

$$
\mathcal{V}_{\beta}^{-}(y) \ll y^{-\frac{5}{4}} \int_{0}^{\infty} x^{-\frac{5}{4}} \phi\left(\frac{x-h}{X}\right) \mathrm{d} x \ll y^{-\frac{5}{4}} X^{-\frac{1}{4}}
$$

and

$$
\begin{aligned}
\mathcal{V}_{\beta}^{+}(y)= & y^{-\frac{1}{4}} \sum_{ \pm} c_{1}^{ \pm} \int_{0}^{\infty} x^{-\frac{1}{4}} \phi\left(\frac{x-h}{X}\right) e(-\beta x \pm 2 \sqrt{x y}) \mathrm{d} x \\
& +y^{-\frac{3}{4}} \sum_{ \pm} c_{2}^{ \pm} \int_{0}^{\infty} x^{-\frac{3}{4}} \phi\left(\frac{x-h}{X}\right) e(-\beta x \pm 2 \sqrt{x y}) \mathrm{d} x+O_{f}\left(y^{-\frac{5}{4}} X^{-\frac{1}{4}}\right) .
\end{aligned}
$$

Changing variable $x \rightarrow X t^{2}$, we have

$$
\begin{aligned}
\mathcal{V}_{\beta}^{+}(y)= & 2 y^{-\frac{1}{4}} X^{\frac{3}{4}} \sum_{ \pm} c_{1}^{ \pm} \int_{0}^{\infty} t^{\frac{1}{2}} \phi\left(t^{2}-\frac{h}{X}\right) e\left(-\beta X t^{2} \pm 2 \sqrt{y X} t\right) \mathrm{d} t \\
& +2 y^{-\frac{3}{4}} X^{\frac{1}{4}} \sum_{ \pm} c_{2}^{ \pm} \int_{0}^{\infty} t^{-\frac{1}{2}} \phi\left(t^{2}-\frac{h}{X}\right) e\left(-\beta X t^{2} \pm 2 \sqrt{y X} t\right) \mathrm{d} x+O_{f}\left(y^{-\frac{5}{4}} X^{-\frac{1}{4}}\right) .
\end{aligned}
$$

Denote $\rho(t)=-\beta X t^{2} \pm 2 \sqrt{y X} t$. If $y>4|\beta|^{2} X$, we have $\left|\rho^{\prime}(t)\right|=|-2 \beta X t \pm 2 \sqrt{y X}| \geq$ $2 \sqrt{y X}-2|\beta| X t \geq 2 \sqrt{y X}-2|\beta| X \geq \sqrt{y X}$. By partial integration twice, we have

$$
\int_{0}^{\infty} t^{ \pm \frac{1}{2}} \phi\left(t^{2}-\frac{h}{X}\right) e\left(-\beta X t^{2} \pm 2 \sqrt{y X} t\right) \mathrm{d} t \ll \Delta y^{-1} X^{-1},
$$

and by (5.5),

$$
\mathcal{V}_{\beta}^{+}(y) \ll_{f} \Delta y^{-\frac{5}{4}} X^{-\frac{1}{4}} .
$$

If $y \leq 4|\beta|^{2} X$, we have $\left|\rho^{\prime \prime}(t)\right|=|-2 \beta X| \gg 1$. By the second derivative test,

$$
\int_{0}^{\infty} t^{ \pm \frac{1}{2}} \phi\left(t^{2}-\frac{h}{X}\right) e\left(-\beta X t^{2} \pm 2 \sqrt{y X} t\right) \mathrm{d} t \ll \frac{1}{\sqrt{|\beta| X}}
$$

and by (5.5),

$$
\mathcal{V}_{\beta}^{+}(y) \ll_{f} y^{-\frac{1}{4}} X^{\frac{1}{4}}|\beta|^{-\frac{1}{2}}+y^{-\frac{3}{4}} X^{-\frac{1}{4}}|\beta|^{-\frac{1}{2}}+y^{-\frac{5}{4}} X^{-\frac{1}{4}} .
$$


By (3.17), (5.4), (5.6) and (5.7), we have

$$
\begin{aligned}
& \sum_{m \geq 1}\left|\lambda_{f}(m)\right|\left|\mathcal{V}_{\beta}^{ \pm}\left(\frac{m}{q^{2}}\right)\right| \\
& \ll_{f} \sum_{m \leq 4|\beta|^{2} X q^{2}}\left|\lambda_{f}(m)\right|\left(\left(\frac{m}{q^{2}}\right)^{-\frac{1}{4}} X^{\frac{1}{4}}|\beta|^{-\frac{1}{2}}+\left(\frac{m}{q^{2}}\right)^{-\frac{3}{4}} X^{-\frac{1}{4}}|\beta|^{-\frac{1}{2}}+\left(\frac{m}{q^{2}}\right)^{-\frac{5}{4}} X^{-\frac{1}{4}}\right) \\
& +\Delta \sum_{m>4|\beta|^{2} X q^{2}}\left|\lambda_{f}(m)\right|\left(\frac{m}{q^{2}}\right)^{-\frac{5}{4}} X^{-\frac{1}{4}} \\
& \ll_{f} \quad q^{\frac{1}{2}}|\beta|^{-\frac{1}{2}} X^{\frac{1}{4}} \sum_{m \leq 4|\beta|^{2} X q^{2}}\left|\lambda_{f}(m)\right| m^{-\frac{1}{4}}+q^{\frac{3}{2}}|\beta|^{-\frac{1}{2}} X^{-\frac{1}{4}} \sum_{m \leq 4|\beta|^{2} X q^{2}}\left|\lambda_{f}(m)\right| m^{-\frac{3}{4}} \\
& +q^{\frac{5}{2}} X^{-\frac{1}{4}} \sum_{m \leq 4|\beta|^{2} X q^{2}}\left|\lambda_{f}(m)\right| m^{-\frac{5}{4}}+\Delta q^{\frac{5}{2}} X^{-\frac{1}{4}} \\
& \ll_{f} \quad q^{2}|\beta| X+q^{2}+\Delta q^{\frac{5}{2}} X^{-\frac{1}{4}} .
\end{aligned}
$$

This proves Proposition 1.

ACKNowledgements. The author is very grateful to the referee for detailed comments and valuable suggestions which bring many improvements on the original draft. This work is supported by National Natural Science Foundation of China (11101239), Young Scholars Program of Shandong University, Weihai (2015WHWLJH04) and Natural Science Foundation of Shandong Province (ZR2016AQ15).

\section{REFERENCES}

[1] V. Blomer, G. Harcos, P. Michel and Appendix 2 by Z. Mao, A Burgess-like subconvex bound for twisted L-functions, Forum Math. 19 (2007), 61-105.

[2] W. Duke, J. Friedlander and H. Iwaniec, Bounds for automorphic L-functions, Invent. Math. 112 (1993), 1-8.

[3] I. S. Gradshteyn and I. M. Ryzhik, Table of Integrals, Series, and Products, Seventh Edition, New York, Academic Press.

[4] G. Harcos, P. Michel, The subconvexity problem for Rankin-Selberg L-functions and equidistribution of Heegner points. II, Invent. Math. 163 (2006), 581-655.

[5] D. R. Heath-Brown, Cubic forms in ten variables, Proc. London Math. Soc. 3 (2) (1983), 225-257.

[6] R. Holowinsky, A sieve method for shifted convolution sums, Duke Math. J. 146 (2009), 401-448.

[7] M. N. Huxley, Area, lattice points, and exponential sums, Oxford University Press, 1996.

[8] H. Iwaniec, Topics in Classical Automorphic Forms, Graduate Studies in Mathematics, vol. 17, Amer. Math. Soc., Providence, 1997.

[9] M. Jutila, Transformations of exponential sums, Proceedings of the Amalfi Conference on Analytic Number Theory (Maiori 1989), Univ. Salerno, Salerno, 1992, 263-270.

[10] H. Kim, Functoriality for the exterior square of $G L_{4}$ and the symmetric fourth of $G L_{2}$, with appendix 1 by D. Ramakrishnan and appendix 2 by H. Kim and P. Sarnak, J. Amer. Math. Soc. 16 (2003), 139-183.

[11] E. Kowalski, P. Michel and J. Vanderkam. Rankin-Selberg L-functions in the level aspect, Duke Mathe. J. 114 (2002), 123-191. 
[12] W. Luo, Shifted convolution of cusp-forms with $\theta$-series, Abh. Math. Semin. Univ. Hambg. 81 (2011), 45-53.

[13] W. Luo and P. Sarnak, Mass equidistribution for Hecke eigenforms, Comm. Pure Appl. Math. 56 (2003), 874-891.

[14] G. Lü, J. Wu and W. Zhai, Shifted convolution of cusp-forms with $\theta$-series, Ramanujan J. (2015), DOI 10.1007/s11139-015-9678-8.

[15] R. Munshi, Shifted convolution sums for $G L(3) \times G L(2)$, Duke Math. J. 162 (2013), 2345-2362.

[16] H.A. Ravindran, On shifted convolution sums involving the Fourier coefficients of theta functions attached to quadratic forms, Phd thesis (2014).

[17] Q. F. Sun, Shifted convolution sums of $G L_{3}$ cusp forms with $\theta$-series, Int. Math. Res. Notices 2017 (6) (2017), 1805-1829.

[18] R. C. Vaughan, The Hardy-Littlewood method, Cambridge University Press, 1997.

School of Mathematics and Statistics, Shandong University, Weihai, Weihai, Shandong 264209, China

E-mail address: qf sun@sdu.edu.cn 